\title{
Étude longitudinale de la prise de notes d'un cours universitaire français : le cas d'étudiants étrangers d'un cursus européen
}

\section{Martine Faraco}

\section{(2) OpenEdition \\ Journals}

Édition électronique

URL : http://journals.openedition.org/asp/2963

DOI : 10.4000/asp.2963

ISSN : 2108-6354

Éditeur

Groupe d'étude et de recherche en anglais de spécialité

\section{Édition imprimée}

Date de publication : 1 décembre 1997

Pagination : 41-54

ISSN : 1246-8185

\section{Référence électronique}

Martine Faraco, «Étude longitudinale de la prise de notes d'un cours universitaire français : le cas d'étudiants étrangers d'un cursus européen », ASp [En ligne], 15-18 | 1997, mis en ligne le 30 avril 2012, consulté le 19 avril 2019. URL : http://journals.openedition.org/asp/2963; DOI : 10.4000/asp.2963

Ce document a été généré automatiquement le 19 avril 2019

Tous droits réservés 


\title{
Étude longitudinale de la prise de notes d'un cours universitaire français : le cas d'étudiants étrangers d'un cursus européen
}

\author{
Martine Faraco
}

1 Le présent travail ${ }^{1}$ a pour objectif de suivre le processus d'évolution de la technique de prise de notes chez les étudiants étrangers du cursus LEA Europe, dans le cadre d'un cours magistral en économie et en droit des affaires en langue française. À la différence de notre dernière enquête transversale (Faraco 1997), l'analyse s'inscrira dans une perspective longitudinale et sera uniquement centrée sur les publics étrangers majoritaires de ce cursus, à savoir espagnols, allemands et anglais, afin d'apporter quelque lumière sur une réalité complexe de la vie universitaire des étudiants étrangers dans leur université d'accueil.

2 L'objectif de ces étudiants est d'approfondir quelques langues cibles (LC) dans une discipline. Ils suivent, la plupart du temps des cursus universitaires de leur spécialité, alors que le cours de soutien linguistique qui leur est dispensé n'aborde que de façon restrictive leurs problèmes de langue. Le programme LEA Europe consiste donc en un apprentissage en immersion des LC de spécialité dans les universités d'accueil. On peut imaginer que leur difficulté majeure est d'envisager les cours en LC et notamment de prendre des notes.

3 Nous voudrions donc savoir s'il est possible de déterminer les facteurs qui influencent cette tâche, quasiment déterminante pour la réussite des études en France. À long terme, sera poursuivie la définition de la teneur d'une aide plus efficace pour la technique de prise de notes par les étudiants bénéficiant de ces échanges européens de sorte que l'acception de «cursus intégrés » puisse être appliquée à ces derniers, de façon optimale (Haeusser 1995). 


\section{Champ d'observation}

4 Pendant trois mois, nous avons participé, avec les étudiants français et étrangers du cursus LEA, aux cours de sciences économiques et juridiques, destinés aux étudiants de $2^{\mathrm{e}}$ année du premier cycle.

$5 \quad$ Les prises de notes effectives des étudiants étrangers, traces écrites d'une compréhension orale, lesquelles constituent nos observables, ont été recueillies en deux étapes : au tout début de l'année universitaire (octobre 1996); au troisième mois de l'année scolaire qui correspond à une période précédant le premier partiel (décembre 1996). Ici, nos étudiants ont suivi une initiation à la technique de prise de notes qui leur a été proposée dans notre cours de soutien linguistique.

6 En raison de la diversité des emplois du temps, nous n'avons pu enquêter auprès de tous les étudiants du cursus LEA Europe. Notre étude porte sur les prises de notes de trois étudiantes anglaises, cinq allemandes et quatre espagnoles ${ }^{2}$. Les étudiantes ont entre 20 et 26 ans.

7 Les données ont ensuite été dépouillées, selon le cours (droit des affaires ou économie) et selon l'origine du groupe linguistique d'appartenance (anglais, allemand, espagnol).

\section{Typologie des enseignants et étudiants}

\subsection{Profil des enseignants}

8 Nous avons observé deux cours de sciences économiques et un cours de droit des affaires. Pour les trois enseignants concernés, nous décrivons sommairement le climat des cours auxquels nous avons assisté.

\section{En sciences économiques}

9 Le plan, bien établi, préalablement diffusé aux étudiants par l'intermédiaire de la plaquette du début d'année, est similaire pour les deux cours d'économie. Cours très vivant, l'enseignant $\mathrm{A}$ travaille en dialogue avec ses étudiants, les questionnant très souvent sur les prérequis ou sur les définitions. Ce cours est étayé par des exemples ou des simulations de processus économiques expliqués en classe. Par ailleurs, A fait une utilisation maximale du tableau: mots clés, schémas, équations, noms propres. En général, A donne une question à préparer ou un point à expliciter ou à approfondir pour le cours suivant. Par ailleurs, A propose aux étudiants de créer un index personnel avec les mots clés assortis de leur définition. Cet index a été amorcé lors du 2e cours.

Les difficultés inhérentes à cette forme de cours semblent résider dans la gestion thématique par les scripteurs non natifs/scriptrices non natives (SNN), qui doivent saisir les thèmes principaux parmi des thèmes secondaires (commentaires, digressions, illustrations). Il s'agit de "séquences latérales ${ }^{3}$ pendant lesquelles l'enseignant explicite un détail ou fait un retour en arrière, par exemple, sur un prérequis nécessaire (non connu ou bien oublié par les étudiants) ou sur quelque point expliqué précédemment. Les SNN doivent être très vigilants pour dissocier ce qui est essentiel de ce qui est secondaire. Le plus souvent, les étudiants étrangers ont du mal à saisir le propos d'autres étudiants natifs qui sollicitent une explicitation de l'enseignant. 
11 Le cours de l'enseignant $B$, articulé sur le même plan que précédemment, est plus traditionnel : il confère à son cours un rythme d'autant plus lent que son exposé est riche en reformulations et pauses. Le discours est d'une grande clarté et les noms propres et les définitions sont dictés. B fait une utilisation minimale du tableau. La participation des étudiants nous a semblé très faible.

\section{En sciences juridiques}

12 Les notes relevées ici sont généralement moins longues que pour les autres cours. L'enseignant C, comme les deux autres enseignants, a diffusé le plan de son cours dans la plaquette. Il affectionne les schémas et le tableau constitue une des pièces maîtresses de sa technique d'enseignement (utilisation de craies de couleurs différentes). Sensible à la compréhension des étudiants, $C$ reformule son explication et interpelle les étudiants pour vérifier s'ils ont compris. Comme A, l'enseignant $C$ instaure un dialogue avec les étudiants, qui, de ce fait, ont tendance à poser beaucoup de questions, et il finit son cours en donnant une question à préparer pour la fois suivante.

\subsection{Profil des étudiants}

Il s'avère ${ }^{4}$ que les anglophones ont appris le français pendant cinq ans au lycée et un an à l'université. Nos informatrices anglaises ont déjà suivi une année de cours dans une université étrangère à Cologne. Elles ont donc fait deux ans d'économie et de droit, un an en langue maternelle (LM) et un an en allemand ${ }^{5}$.

Pour les Espagnoles (trois ans de français au lycée et un an à l'université), le cursus français est leur premier séjour dans une université étrangère. Elles ont fait un an de droit et un an d'économie à l'université de Grenade en LM.

Il en va de même pour les Allemandes (sept ans de français au lycée et de un à quatre ans à l'université), pour qui cette année correspond à la première expérience dans une université étrangère. Elles n'ont suivi qu'un an de cours de droit et d'économie en LM.

\section{Réflexion métacognitive sur la prise de notes en LM et en LC}

16 À l'instar de Noël $(1991,1995)$ et Noël et al. $(1995,1996)$, nous nous intéressons au recul que prennent les étudiants par rapport à leur travail de prises de notes. Ainsi, comme préliminaire à notre analyse, il nous a semblé pertinent d'examiner quelques témoignages des étudiants quant à leur tâche de prises de notes. Voici quelques réponses écrites ${ }^{6}$ à la question « Dites ce qui vous semble différent entre la façon dont vous prenez des notes en français et en LM ».

Allemandes :

- En France, on utilise plus d'abréviations qu'en Allemagne. Il y a seulement quelques mots importants qui sont abrégés par tout le monde. Mais en général, on n'est pas habitués à faire des abréviations en Allemagne.

- En langue maternelle : pas habitué à utiliser des abréviations.

1. ce n'est pas nécessaire

2. on arrive plus facilement à relire ce qu'on a écrit.

- En allemand, je ne suis pas habituée à utiliser des abréviations ; les mots se terminent d'une façon différente chaque fois, seules abréviations (isch(en), je 
n'ai pas besoin d'écrire des phrases entières ; en français, j'écris presque tout ce que le prof dit, sinon j'ai peur de ne pas le comprendre plus tard.

- En français, on utilise des signes pour abréger $\left({ }^{\circ}, t\right)$ et en Allemagne, on écrit le mot à moitié et puis on met un point.

Espagnoles :

- Je ne crois pas qu'il y ait des grandes différences, mais comme en français, j'ai toujours appris à mettre I, II... je continue à le faire. En espagnol, par contre, cette technique n'est pas très utilisée si ce n'est pour développer une idée où l'on mettra $1,2,3 \ldots$ pour les différentes sous-idées ou caractéristiques de l'idée.

Anglaises :

- Je ne me rappelle plus comment je prends les notes en anglais, ça fait longtemps que je n'ai rien fait en anglais. En français, je prends les notes n'importe comment, je m'en fiche si j'ai fait des fautes d'orthographe - ce qui est important est d'avoir une phrase qu'on peut comprendre après. J'utilise souvent toutes les 3 langues pour pouvoir mieux et plus vite écrire. J'ai développé un système et je crois que j'arrive bien - on verra après les examens.

- En français, j'utilise pour la plupart les abréviations correspondantes anglaises. Toujours « e.g. » par exemple. Pour les choses importantes*. Mais j'essaie d'écrire tous les mots en plein en Français donc je n'ai pas de problèmes quand je les relis. Il n'y a pas de différences entre ma façon de prendre des notes en français et en anglais, sauf que j'utilise plus les abréviations quand j'écris en anglais.

\section{Résultats de l'enquête}

17 Nous avons catégorisé, lors de la précédente enquête (Faraco 1997) les différents éléments attendus pour une bonne compréhension de ce cours : séquence d'ouverture et de clôture du cours ou des différentes parties du cours ; idées importantes ; lisibilité des mots clés ; transitions assurant la continuité de la logique de l'exposé; structure; abréviations (courantes, utilisées pour gagner en vitesse); fréquence d'utilisation desdites abréviations; familiarité et aisance d'utilisation des sigles spécifiques d'économie; explicitation systématique des sigles nouveaux ; incompréhension signalée ; recours à la LM pour les étudiants étrangers.

18 Par ailleurs, étant données les contraintes de l'enquête (voir supra, 2.1.) et étant donné que les caractéristiques semblent suffisamment récurrentes à l'intérieur d'un groupe linguistique, nous décidons d'articuler le dépouillement des données de la façon suivante : traitement conjoint des deux disciplines (droit des affaires et économie) et traitement disjoint des trois groupes linguistiques (espagnol, anglais et allemand).

\subsection{Premier dépouillement (octobre 1996)}

\subsubsection{Les abréviations}

Pour les trois groupes, les mots longs en «- ment» et «- tion » et les mots usuels sont généralement retranscrits en entier: opération, thésaurisation, habitation développement, établissement, point de vue général, société, économie, toute... 

germanophones, tandis qu'elles sont bien présentes chez les anglophones. Ces dernières, en outre, les utilisent sans explication. Pour justifier ce degré de familiarité qui semble apparaitre, on peut invoquer le fait que les étudiants anglaises ont fait deux ans d'économie (voir supra 2.2.) et, par conséquent, manient plus aisément les abréviations économiques classiques.

$$
\begin{aligned}
& \text { S. <- save (épargne) } \\
& \text { W. <- work (travail) }
\end{aligned}
$$

Sont adoptées par tous les étudiants les abréviations spécifiques à la discipline, proposées par l'enseignant. À noter que la transcription complète des abréviations (entre parenthèses) a été notée par toutes les étudiantes sauf par les anglaises. Familiarité ou manque de temps pourraient être des facteurs explicatifs :

\section{S.Q.S.N.F. (Sociétés ou Quasi Sociétés Non Financières)}

F.B.C.F. (Formation Brute de Capital Fixe)

L'absence d'abréviations, constatée généralement, se traduit dans les prises de notes par des répétitions gratuites. Nous trouvons sous la plume d'une germanophone :

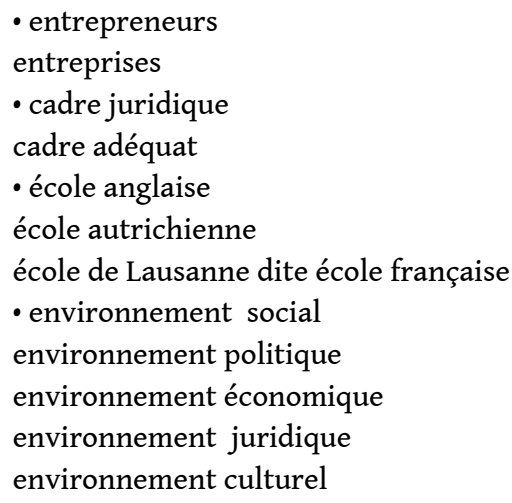

ou chez une hispanophone :

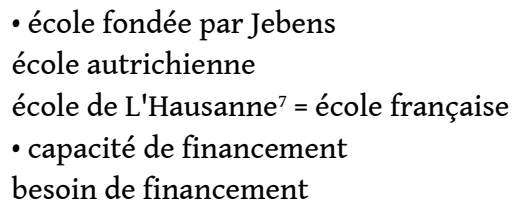

Quand il apparaît des abréviations (ce qui est un cas beaucoup plus rare que le précédent), les Anglaises utilisent des «troncations ", c'est-à-dire que seules les premières lettres du mot sont reproduites. Ce phénomène est amplifié pour les Allemandes. À la relecture, cela peut provoquer des confusions puisqu'en français, de nombreux mots commencent de la même façon, mais se terminent de façon différente :

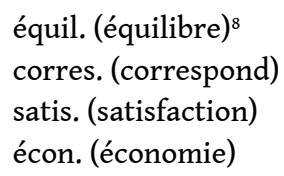

Quand il n'y a pas d'ambiguïté, seule la première lettre du mot apparait chez les Allemandes. Ce type d'abréviations est toujours « contextuel»: 


$$
\begin{aligned}
& \text { v. d'échange (valeur) } \\
& \text { le m. se développe (marché) }
\end{aligned}
$$

Quand le terme revient plusieurs fois dans les propos de l'enseignant, les étudiants sont en mesure d'utiliser les abréviations de façon évolutive (néoclassique $\rightarrow$ néoclass), mais ils montrent une hésitation entre deux termes (néoclassique $\longrightarrow \rightarrow$ néoclass ou $\mathrm{S}<->$ Sté). Nous y distinguerons des traces d'« évolution »'et des traces d'« instabilité ».

En somme, tous les groupes linguistiques suivent la même logique: pas ou peu d'abréviations et de timides essais avec troncations, abréviations contextuelles ou encore traces d'évolution et d'instabilité.

7 Cependant, nous remarquons que les abréviations utilisées par les Espagnoles ressemblent davantage à celles qu'utilisent les Français: mots finissant par «-tion»: liquidat $^{\circ}$ (liquidation), format ${ }^{\circ}$ (formation)... mots finissant par «-ment » : enrichissemt (enrichissement), seulema, (seulement)... Sans doute faudrait-il expliquer ce phénomène par la transparence linguistique entre le français et l'espagnol. En outre, pour ce groupe linguistique, sont également observées des abréviations de structure plus complexe visant à une certaine systématisation ou/et à la levée d'ambiguïté : ^ $\mathrm{m}$ (même), ense (entreprise)... En tout cas, aisée et très fréquente chez les natifs, cette pratique de l'abréviation reste relativement malaisée et sporadique chez les SNN.

\subsubsection{Structure logique}

8 Les SNN comprennent, en général, l'articulation globale du cours, puisque «l'horizon » $\mathrm{du}$ cours leur est connu. En revanche, certaines parties, quelque peu autonomes, ne peuvent pas être directement replacées dans une perspective globale et, par conséquent, la SNN n'en dégage pas bien la logique. Certaines séquences n'apparaissent pas du tout comme l'explicitation de la différence des termes objectif, finalité ou mission et but, explicités en droit (ce passage est absent dans les notes d'une anglophone). De même, des parties historiques ou récapitulatives deviennent une «mosaïque » de mots juxtaposés avec, parfois, des flèches en guise d'articulation logique, flèches favorisant peu la compréhension puisque polyvalentes ${ }^{10}$. Dans la prise de notes du cours d'économie d'une étudiante anglaise, quelques mots sont alignés sur deux colonnes (voir tableau 1).

Tableau 1

\begin{tabular}{|l|l|}
\hline mercantilisme & XVIIIe s. libéralisme \\
XVIe $\rightarrow$ XVIIIe & recherche d'un principe de régulation \\
métaux précieux & Smith la main invisible \\
inflation & Société \\
$====>$ individus & \\
\hline
\end{tabular}

Voici ce qui devait être compris ${ }^{11}$ :

Du XVI ${ }^{e}$ au XVIII ${ }^{e}$, la réflexion s'est autonomisée. Jusqu'alors la société fonctionnait sur le mercantilisme: la richesse du royaume dépendait de la richesse du monarque en métaux précieux. Deux attitudes complémentaires: protéger le marché extérieur et être agressif à l'exportation. Du XVI ${ }^{e}$ au XVIII ${ }^{e}$, il s'agit d'une période de conflits permanents. Ex. : l'inflation en Espagne à la fin du XVI ${ }^{e}$ montre 
que l'intérêt de l'individu n'est pas forcément conforme à l'intérêt du monarque. D'où une nouvelle idée : la richesse doit procéder des individus.

Ceci va se confirmer au XVIII ${ }^{\mathrm{e}}$ siècle au moment où va naître le libéralisme, doctrine qui va supplanter le mercantilisme. On va envisager l'équilibre social par le jeu de la somme des individus. Il y a principe de régulation alternatif. A. Smith $(\mathrm{XVIII})^{e}$ va être le principal moteur de ce courant libéraliste. Il isole les principes (rudiments) de la micro-économie de base : « la main invisible ». Comment chacun peut-il poursuivre son intérêt mais sans déboucher sur le conflit généralisé ? On prend une société quelconque composée d'individus : souverains (maîtres de leur choix), rationnels, hédonistes. La « main invisible » c'est un mécanisme des prix, de flexibilité des prix qui va s'autoréguler. linguistique d'appartenance. En général, la SNN l'indique par un point d'interrogation. Toutefois, ces problèmes semblent peu prédictibles puisque, même les Espagnoles, considérées comme les étudiantes du groupe les plus avancées linguistiquement, peuvent commettre un contresens, rencontré par ailleurs chez les Allemandes : la main visible pour la main invisible. Les SNN sont confrontées à un problème de segmentation de la chaîne verbale, dû certainement à une sous-articulation de l'enseignant C (voir supra, 2. 1) ou à son recours minimal au tableau. Dans d'autres cas, il peut s'agir d'une non-compréhension du contexte ou encore, à l'apparition dans les propos de l'enseignant, d'un mot ou d'une structure non disponible dans l'interlangue (IL) du SNN. Une Allemande en est un exemple :

contrefacteur (contrefaçon)

gérir (gérer)

\subsubsection{Code switching}

Le code switching (ou alternance codique) est un terme de sociolinguistique employé par Fishman (1971). Calvet (1993 : 29) en rappelle la définition :

Lorsqu'un individu est confronté à deux langues qu'il utilise tour à tour, il arrive qu'elles se mélangent dans son discours ${ }^{13}$ et qu'il produise des énoncés 
«bilingues ». Il ne s'agit plus ici d'interférence mais, pourrait-on dire, de collage, du passage en un point du discours d'une langue à l'autre, que l'on appelle mélange de langues (sur l'anglais code mixing) ou alternance codique (sur l'anglais code switching ), selon que le changement de langues se produit dans le cours d'une même phrase ou d'une phrase à l'autre ${ }^{14}$

ou en droit :

Entreprise = has no legal rights ??? ${ }^{16}$ [= n'a pas de droits légaux] So that mère ${ }^{17}$ has majority she must have more than $50 \%$ of the capital [= pour que la société mère soit majoritaire, elle doit avoir plus de $50 \%$ du capital]

\subsection{Second dépouillement (fin décembre 1996)}

\subsubsection{Les abréviations}

Les trois groupes linguistiques continuent à conserver au fil de leurs prises de notes, des mots longs en «-ment » et en «-tion » non abrégés. On peut voir cette tendance s'accuser ou s'estomper suivant le groupe linguistique observé. Cette pratique se trouve être en concurrence avec une tendance à l'utilisation des abréviations conventionnelles. De temps à autre, le noyau vocalique est moins stable que dans une abréviation française. Une des anglophones écrit : 


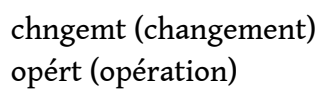

En revanche, certaines SNN allemandes persistent à ne pas utiliser d'abréviations pour les mots longs et l'on trouve le mot rendement écrit à la suite deux fois en entier.

Les traces d'abréviations se caractérisent, en effet, par complexité et manque d'économie. Les Anglaises utilisent essentiellement la troncation et l'abréviation contextuelle qui peut se réduire parfois à la première lettre du mot. Peu de mots usuels sont raccourcis. L'instabilité reste apparente.

41 Par rapport à la première observation, les Allemandes ont appris à abréger de préférence les mots usuels aux mots longs (voir tableau 2).

Tableau 2

\begin{tabular}{|l|l|}
\hline st & sont \\
\hline$\wedge^{\mathrm{m}}$ & même \\
\hline à condition q & à condition que \\
\hline ê & être \\
\hline 1 & un \\
\hline qc & quelque chose \\
\hline
\end{tabular}

Les signes mathématiques deviennent également plus fréquents :

/ ou \(croissance ou baisse)

ou $<$ (inférieur ou supérieur)

Ces SNN dissocient l'abréviation dans les formules $C$ (coût) et dans le texte coût ${ }^{18}$.

Ce sont les hispanophones qui montrent le moins de stabilité: abréviation et nonabréviation des mots; un grand nombre de troncations, racine + dernière lettre ou dernière syllabe; distorsion orthographique abrégeante (provoké, dynamic, organ); recours aux signes mathématiques; quelques mots usuels; dissociation du texte et des formules.

\subsubsection{Structure logique}

Elle n'est pratiquement reflétée par aucun articulateur du discours. Il faut peut-être dire que les matières ou les techniques d'enseignement de tel ou tel enseignant (par ex. B) s'y prêtent peu. À l'exception de deux étudiantes qui continuent à "écrire au kilomètre ", toutes nos informatrices, insérant par endroits, des tirets pour indiquer une idée nouvelle, ont le même type de comportement organisateur: retraits, soulignements pleins ou en pointillés, encadrés, parenthèses.

47 Une singularité, cependant, à noter chez les Allemandes est l'utilisation de la LM à des fins de structuration (voir infra 4.2.4). Les Espagnoles, quant à elles, persistent dans leur 
pratique de l'«enregistrement» écrit de l'intégralité des propos de l'orateur sans structuration personnelle de leur part, ici en économie :

En réalité, en courte période, l'ense pourra produire même si le prix de marché est inférieur au CM (coût moyen) à condition que ce prix soit supérieur au CVMe (coût variable moyen), qui est le seuil de fermeture. En courte période l'ense a des prix fixes même si la product $^{\circ}$ est nulle. Elle va essayer de voir si en produisant elle perd encore moins que si elle ne produit rien du tout [...]

\subsubsection{Compréhension}

Les problèmes de compréhension semblent avoir pratiquement disparu chez toutes les SNN, quel que soit leur groupe linguistique d'appartenance, le point d'interrogation, signalant la difficulté, en début d'année, est absent. Peut-être cela peut-il encore se reproduire cependant, témoins ces quelques exemples, dont le dernier témoigne d'une mauvaise segmentation persistante, dans les notes d'une anglophone :

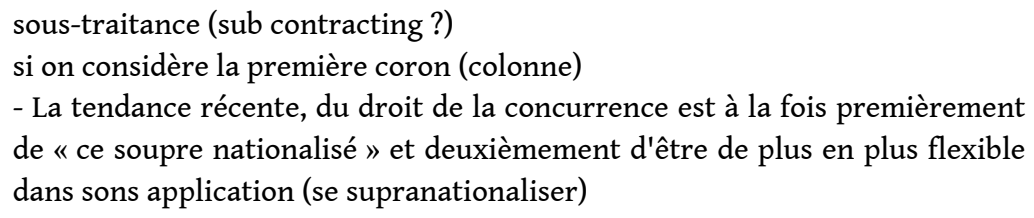

\subsubsection{Code switching}

Le recours à la LM ne s'observe plus chez les Espagnoles. Par contre, cela semble s'être accru et systématisé pour les deux autres groupes. Les germanophones traduisent souvent les mots clés :

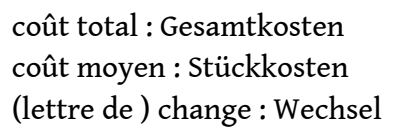

50 De façon plus remarquable, ces SNN en usent à des fins de «structuration métadiscursive ${ }^{19}$ ", caractérisée par la prise de distance par rapport au plan direct du contenu du cours. De la sorte, la LM peut attribuer aux différentes parties une étiquette plus générale :

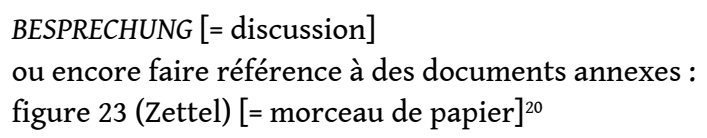

51 Comme dans les premières observations, ce sont les Anglaises qui utilisent presque massivement la LM pour rendre compte des explications que donne l'enseignant. En effet, en raison du rythme trop rapide de ces séquences latérales, les anglophones préfèrent la traduction interlinguale à celle intralinguale, leur demandant un coût cognitif plus important. Ce système de code switching, visible déjà lors du premier recueil de données, semble s'être accentué et systématisé.

- monopole contrarié : collusion could happen [= une collusion pourrait avoir lieu]

pot de vin - bribe other not to take the contract [= soudoyer quelqu'un pour qu'il ne signe pas le contrat] --> cartellisation d'offre - collusion concurrence imparfaite all not on the table [= tout n'est pas clair] au terme du processus (above) [= voir plus haut] 
- « pot de vin » - but firms have collided so each firm gets a chance to fulfill a contract [ = mais les firmes sont entrées en concurrence de sorte que chaque firme a une chance d'emporter un contrat]

Oligopole pur: Few big firms ---> lot of small firms to fulfill orders [= quelques grandes firmes ---> un grand nombre de petites firmes pour satisfaire des commandes]

Darwin ---> survival of the fittest [= survie des plus forts]

l'abus de position dominante 1890 - named by Americans as «SHERMAN Act » [= appelé par les Américains « SHERMAN Act »]

Dans tous les pays développés, il existe des réglementations de ce type (monopole de fait) en Europe aussi (anti-trust). défini par l'article $85 \mathrm{du}$ traité du Rome 25 mars 1937. If blocking entry to the market [= Si on bloque l'entrée du marché]

les faits : orders of dates + what happened, tell story with only facts [= ordre chronologique + ce qui s'est passé, racontez l'histoire avec seulement les faits]

indefiniblement endossable sign on back and pass on to other people [= signe au dos et passent aux autres personnes]

by signing shows that he did receive goods [= le fait qu'il a signé montre qu'il a reçu du matériel]

In France at banks : list of people who have not honoured their credit notes to make people aware [= En France dans les banques : liste de personnes qui n'ont pas honoré leurs notes de crédit pour rendre les gens vigilants]

\section{Conclusion} d'année n'utilisent pratiquement pas d'abréviations. Ceci entraine un décalage entre le discours de l'enseignant et leur prise de notes. Par là même, le rythme n'étant pas soutenu la logique des parties explicatives ou séquences latérales ne peut être conservée. Les SNN recourent donc souvent à la LM. D'une manière générale la compréhension en souffre et il est possible d'inférer que la révision pour les partiels sera d'autant plus difficile.

de notes, les SNN se distinguent encore les uns des autres, en fonction de leur groupe linguistique d'appartenance. En concurrence avec des abréviations de SN non encore maîtrisées, ils ont développé un système qui leur est propre. Instabilité et manque d'économie restent encore les caractéristiques essentielles de cette prise de notes en LC. La mise en page de leurs notes écrites, au bout de quelques mois, prend efficacement le relais de la structure logique. La LM, absente chez les Espagnoles, est un substitut efficace à leurs défaillances en LC pour les Anglaises et un outil de structuration métadiscursive pour les Allemandes. Malgré la non-systématicité de la prise de notes des SNN, nous pouvons observer, dès à présent, un gain de rapidité et donc une diminution des 
problèmes de compréhension et des lacunes dans leur mise en mots des propos de l'enseignant.

Nous souhaitons familiariser plus rapidement le prochain groupe d'étudiants de cursus européen aux abréviations, généralement utilisées par les SN et les sensibiliser à la nécessité de créer leur propre système d'abréviations. En outre, il nous semble qu'un travail conjoint entre les enseignants de spécialités et les enseignants de soutien linguistique pourrait être pertinent. Ainsi ces derniers, après une initiation aux disciplines telles que le droit ou l'économie, pourraient-ils travailler, dans des groupes restreints, au décryptage des discours oraux en vue de maximiser la prise de notes des étudiants étrangers, tout en poursuivant l'amélioration de la compétence linguistique.

Par ailleurs, cette étude signale que certaines différences, relatives à la disparité des habitudes universitaires européennes, à l'influence de la LM et à l'état de l'IL des étudiants, restent encore à prendre en compte.

Nous souhaitons remercier très chaleureusement Messieurs Weiler, Bassoni et Cazorla, enseignants à l'Université de Provence. Tous trois se sont prêtés à l'expérience avec enthousiasme et ont montré un profond désir d'aider les étudiants étrangers du cursus Europe.

\section{BIBLIOGRAPHIE}

Calvet, L-J. 1993. La sociolinguistique. Coll. Que sais-je ?. Paris : Presses Universitaires de France.

Faraco, M. 1997. «Étude comparative des techniques de prise de notes par les étudiants français et les étudiants étrangers du cursus LEA ». Le Français dans le Monde 287, 38-40.

Fishman, J. 1971 [1969]. Introduction à la sociolinguistique. Bruxelles/Paris : Labor/Nathan.

Haeusser, C. 1995. «Réflexions sur les limites d'une intégration langues-savoirs disciplinaires entre milieux étrangers ». ASp 7-10, 285 - 301.

Noël, B. 1991. La métacognition. Bruxelles : De Boeck.

Noël, B. 1995. « La métacognition. L'art d'évaluer ses performances ». Sciences humaines 56, 23-25.

Noël, B., M. Romainville et J. Wolfs. 1995. « La métacognition : facettes et pertinence du concept en éducation ». Revue française de pédagogie 112, 47-56.

Noël, B., M. Romainville et J. Wolfs. 1996. « Métacognition et prise de notes ». Éduquer et former 5-6.

\section{NOTES}

1. Cette recherche nous a été confiée par la section LEA (Langues Étrangères Appliquées), filière Europe de l'Université de Provence. Ces étudiants poursuivent leurs études universitaires dans deux pays étrangers différents et sont donc amenés à suivre les cours universitaires à l'étranger au même titre que les étudiants du pays d'accueil. Je voudrais remercier Christiane Haeusser 
pour sa lecture de la première version de ce travail, ainsi que ses réactions et conseils. Bien entendu les lapsus et les erreurs subsistant dans ce texte m'appartiennent entièrement.

2. Il faut insister sur le fait que cette étude, étant donné le caractère restreint de l'échantillon, ne peut indiquer que des tendances qu'il s'agira par la suite de vérifier avec un plus grand nombre d'étudiants.

3. Dans la situation exolingue (en classe de langue, par exemple), la séquence latérale est un moment d'explicitation où se pratique un réajustement de la compréhension entre partenaires de compétences linguistiques dissymétriques. En cours de spécialité, il s'agit de dissymétrie de compétence linguistique doublée de connaissances spécifiques de la discipline concernée.

4. Pour l'année universitaire 1996-97, le groupe de LEA Europe est constitué de 24 étudiants : 7 espagnoles, 3 anglaises et 14 allemands.

5. Ces étudiants ont au minimum deux langues cibles.

6. Quand elles n'étaient pas pertinentes pour notre travail de recherche, nous avons rectifié les erreurs orthographiques, sur l'ensemble des corpus.

7. Voir infra 4.1.3. pour les problèmes de compréhension, liés à une difficulté de segmentation de la chaîne parlée.

8. Dans les exemples, à côté de ce qu'a écrit le SNN, figure entre parenthèses le mot auquel l'abréviation renvoie.

9. Cette trace d'une abréviation en évolution est présente chez tous les groupes avec une moindre utilisation chez les étudiantes espagnoles.

10. Dans l'exemple qui suit, la flèche renvoie à deux significations : dans « XVI ${ }^{\mathrm{e}}->\mathrm{XVIII}^{\mathrm{e}}$ ", elle indique un laps de temps; dans «==> individus ", elle indique une provenance.

11. Nous avons intégralement noté, dans les deux paragraphes suivants, ce que le professeur a dit et nous avons mis en gras les mots qui apparaissent dans la prise de notes de l'étudiante.

12. L'une d'entre elles note soigneusement tous les articulateurs.

13. Calvet fait référence ici au discours oral mais nous appliquerons cette remarque au cas de la prise de notes à l'écrit.

14. Dans cette étude, pour simplifier le raisonnement, nous décidons de ne pas faire la distinction entre code mixing et code switching et d'utiliser indifféremment le dernier terme pour désigner ce changement de code.

15. La traduction française, non visible dans la prise de notes mais explicitée par les sujets lors d'un entretien ultérieur, sera indiquée, autant que faire se peut entre crochets carrés et précédée du signe égal.

16. Se reporter aux problèmes de compréhension (voir supra 4.1.3).

17. Société mère

18. Ce mot est revenu un grand nombre de fois ce jour-là dans le cours d'économie, il a été chaque fois écrit en entier.

19. Ou méta-textuelle.

20. La figure se trouve sur un petit papier distribué par l'enseignant.

\section{RÉSUMÉS}

Cette analyse de la prise de notes dans le cadre d'un cours universitaire français s'inscrit dans une perspective longitudinale et est centrée uniquement sur des publics espagnol, allemand et 
anglais, dénommés ici scripteurs non natifs (SNN). Nous nous intéressons aux abréviations, aux problèmes de structuration et de présentation, au recours éventuel au code switching, et nous nous attachons à voir s'il existe des récurrences en fonction du groupe linguistique dont sont issus les étudiants étrangers concernés.

This study of note-taking, within the framework of French academic lectures, is part of a longitudinal perspective and is only focused on Spanish, German and English audiences, named here as non-native writers (NNW). We are interested in abbreviations, problems of structuration and presentation with a possible recourse to code switching, and we try to discover if there exist common recurrences among the linguistic groups of foreign students.

\section{INDEX}

Keywords : abbreviation, code switching, European exchange, interlanguage, note-taking, language acquisition

Mots-clés : abréviation, acquisition des langues, alternance codique, échange européen, interlangue, prise de notes

\section{AUTEUR}

\section{MARTINE FARACO}

Martine Faraco enseigne le français aux étudiants étrangers. Elle est attachée au Service commun d'enseignement du français aux étudiants é trangers (SCEFEE) et à la section LEA de l'Université de Provence. Elle est docteur ès lettres (phonétique) et l'auteur de plusieurs articles de linguistique appliquée sur l'acquisition d'une langue seconde. martine.faraco@univ-provence.fr 